\title{
Effects of $\boldsymbol{H}$ pylori therapy on erythrocytic and iron parameters in iron deficiency anemia patients with $H$ pylori-positive chronic gastristis
}

\author{
Lun-Hua Chen, He-Sheng Luo
}

\author{
Lun-Hua Chen, Department of Hematology, Renmin Hospital of \\ Wuhan University, Wuhan 430060, Hubei Province, China \\ He-Sheng Luo, Department of Gastroenterology, Renmin \\ Hospital of Wuhan University, Wuhan 430060, Hubei Province, \\ China \\ Correspondence to: He-Sheng Luo, Department of Gastro- \\ enterology, Renmin Hospital of Wuhan University, no. 238 Jiefang \\ Road, Wuhan 430060, Hubei Province, \\ China.clh97318@sina.com \\ Telephone: +86-27-88041911213 Fax: +86-27-88042292 \\ Received: April 10, 2007 \\ Revised: August 3, 2007
}

efficacy of ferrous succinate therapy in IDA patients with H pylori-positive chronic gastritis.

(c) 2007 WJG. All rights reserved.

Key words: $H$ pylori; Iron deficiency anemia; Oral iron treatment; Chronic gastritis

Chen LH, Luo HS. Effects of $H$ pylori therapy on erythrocytic and iron parameters in iron deficiency anemia patients with $H$ pylori-positive chronic gastritis. World J Gastroenterol 2007; 13(40): 5380-5383

http://www.wjgnet.com/1007-9327/13/5380.asp

\section{INTRODUCTION}

Spontaneous iron excretion in adults is minimal and iron deficiency anemia (IDA) is generally attributed to abnormal blood $\operatorname{loss}^{[1]}$. IDA is often caused by gastrointestinal bleeding due to peptic ulcer and chronic gastritis, or in women of reproductive age by increased menstrual flow and the greater requirements of pregnancy. Recently, several seroepidemiological studies have suggested a link between IDA and $H$ pylori infection ${ }^{[2,3]}$. A high percentage of $H$ pylori-positive IDA patients exhibit atrophic changes in the gastric body, and the remainders have chronic superficial gastritis extending to the fundic mucosa.

$H$ pylori infection has even been implicated as a cause of IDA refractory to oral iron treatment ${ }^{[4,5]}$. Some patients with refractory IDA have no gastrointestinal symptoms but $H$ pylori gastritis, as the only cause of their anemi $a^{[6]}$. Most dietary iron is in the non-hemic ferric form, and an acidic intragastric $\mathrm{pH}$ is needed to reduce it to the ferrous form for absorption. This reaction is promoted by gastric acidity and ascorbic acid (AA), which is thus considered the most potent regulator of iron absorption ${ }^{[7]}$. Patients with $H$ pylori gastritis showed an increase in intragastric $\mathrm{pH}$ with a median of $>3$, a pH that is known to be critical in the process of iron absorption. Moreover, AA is actively secreted from plasma to the gastric juice ${ }^{[8]}$, but the concentration of AA in the gastric juice of patients with $H$ pylori gastritis and IDA is clearly reduced in comparison with both healthy and nonanemic $H$ pylori-positive controls ${ }^{[0-11]}$.

The availability of convenient, non-invasive methods for identifying $H$ pylori gastritis has greatly facilitated the recognition of infected patients, resulting in progressive 
awareness of its influences and possible role in the causation of $\mathrm{IDA}^{[12]}$. Therefore, we first examined the prevalence of $H$ pylori infection and serum markers of iron deficiency. We then evaluated the effects of subsequent $H$ pylori eradication on the response to oral iron therapy, which could provide valuable information for further clinical applications.

\section{MATERIALS AND METHODS}

\section{Patients}

From January 2002 to December 2005, 86 IDA adult patients with $H$ pylori-positive chronic gastritis were enrolled. The participants comprised 36 women and 50 men; median age 53 years, range 18-76. According to Sydney System, the patients were diagnosed as having atrophy, intestinal metaplasia, or antral versus body gastritis, in 34, 27, and 25 cases, respectively. According to the criteria ${ }^{[13]}$, IDA was defined as hemoglobin $(\mathrm{Hb})$ concentration $<120 \mathrm{~g} / \mathrm{L}$ in the men and $<110 \mathrm{~g} / \mathrm{L}$ in the women, serum ferritin $(\mathrm{SF})<12 \mu \mathrm{g} / \mathrm{L}$, mean corpuscular hemoglobin $(\mathrm{MCH})<27 \mathrm{pg}$, and mean corpuscular volume $(\mathrm{MCV})<80 \mathrm{fL}$. Obvious causes of blood loss, such as active gastrointestinal hemorrhage, menometorrhagia or heavy menstrual loss, and any other non-gastrointestinal disease likely to cause IDA, were exclusion criteria for this study. Also excluded from the study protocol were patients with previous gastric surgery, and those who received anti- $H$ pylori treatment or anti-secretory drugs before. Patients who were lactating or pregnant and those with malnutrition or cancer were also excluded.

\section{Methods}

The ${ }^{14} \mathrm{C}$-urease breath test was performed as described previously ${ }^{[14]}$. Briefly, the patients fasted overnight and were then given urea labeled with $37 \mathrm{kBq}$ of ${ }^{14} \mathrm{C}$ dissolved in water. Breath samples were collected before and 15 min after ingestion, when enzymolysis of labeled urea occurred and ${ }^{14} \mathrm{CO}_{2}$ had been released into the peripheral circulation. The ${ }^{14} \mathrm{CO}_{2}$ in these samples was trapped in 1 $\mathrm{mmol} / \mathrm{L}$ of hyamine hydroxide in ethanol and transferred into scintillation liquid. The ${ }^{14} \mathrm{C}$ content was measured in $\mathrm{Bq}$ mode using a liquid $\beta$-scintillation counter (Headway, China). Samples with $<3.33 \mathrm{~Bq}$ were considered negative for $H$ pylori, while samples with $>3.33 \mathrm{~Bq}$ were considered positive. The peripheral hemogram including $\mathrm{MCH}$ and MCV was measured with a Sysmex automated analyzer (Kobe, Japan). Biochemical assays for serum iron (SI) and $\mathrm{Hb}$ were performed as routine. SF was determined with a chemiluminescent immunometric analyzer and kit from Bayer Co. (Germany).

The 86 patients were randomly divided into two groups of equal size. Group A comprised 43 cases and received oral iron treatment combined with triple therapy for $H$ pylori eradication, while the 43 cases in group $\mathrm{B}$ received oral iron treatment only as controls. Oral iron treatment comprised ferrous succinate (with an $\mathrm{Fe}^{2+}$ content of $34 \%-36 \%$ ) $200 \mathrm{mg}$ supplemented with ascorbic acid 100 $\mathrm{mg}$ three times daily. Treatment was given until the SF recovered to normal. Triple therapy for $H$ pylori eradication included deutero-bismuth citrate $240 \mathrm{mg} \times 2 / \mathrm{d}$, amoxicillin $500 \mathrm{mg} \times 2 / \mathrm{d}$, and metronidazole $400 \mathrm{mg} \times 2 / \mathrm{d}$ for 2 wk; in all cases, $H$ pylori was eradicated.

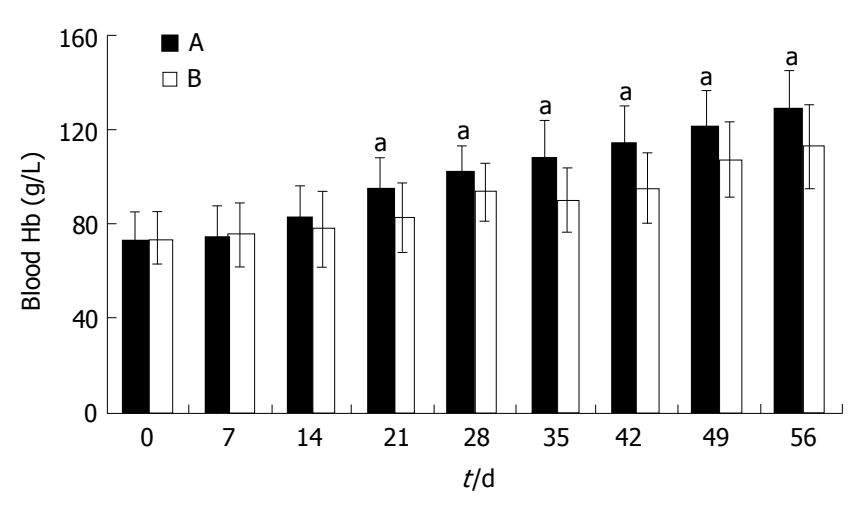

Figure 1 Blood $\mathrm{Hb}$ during iron treatment (mean $\pm \mathrm{SD}, n=43 ;{ }^{\mathrm{a}} P<0.05$ vs group $\mathrm{B}$ ).

\section{Follow-up}

The mean $\mathrm{Hb}, \mathrm{MCH}$, and MCV in the peripheral blood of the two groups were recorded before and at $\mathrm{d} 0,7,14,21$, $28,35,42,49$, and 56 after iron treatment. SI and SF were compared before and at $\mathrm{d} 0,14,28,42$, and 56 .

\section{Statistical analysis}

Each assay was made at least three times and data were expressed as the mean $\pm \mathrm{SD}$. The differences between groups were determined using $t$ test for paired analysis. $P<0.05$ was considered statistically significant.

\section{RESULTS}

\section{Influence of $\mathrm{H}$ pylori on $\mathrm{Hb}$}

$\mathrm{Hb}$ was slightly higher in group A at d 14 after the start of triple therapy for $H$ pylori eradication, but the difference was not significant $(P>0.05)$. After the complete course of triple therapy, the increase of $\mathrm{Hb}$ in group A became significantly faster than that in group B $(P<0.05)$. At d 56, the mean $\mathrm{Hb}$ in group $\mathrm{A}$ returned to the normal level referred to the criteria. In group $\mathrm{B}$, the mean $\mathrm{Hb}$ had also increased compared with that before oral iron treatment, but it was still lower than that in group $\mathrm{A}(P<0.05$, Figure 1$)$.

\section{Influence of $\mathrm{H}$ pylori on $\mathrm{MCV}$ and $\mathrm{MCH}$}

Similar to the trend seen for $\mathrm{Hb}$, the increase of MCV and $\mathrm{MCH}$ in group A was not significantly greater than that in group $\mathrm{B}(P>0.05)$. At $\mathrm{d} 21$, the $\mathrm{MCV}$ and $\mathrm{MCH}$ in group $A$ became normal, and were much higher than those in group $\mathrm{B}(P<0.05)$. In group $\mathrm{B}$, the $\mathrm{MCV}$ and $\mathrm{MCH}$ remained at lower than normal levels until $42 \mathrm{~d}$ after the start of therapy. At this time, the MCV and MCH reached a plateau in both groups and the differences disappeared $(P>0.05$, Figures 2 and 3$)$.

\section{Influence of $\mathrm{H}$ pylori on iron status}

There were no significant differences in parameters of iron status between the two groups at the start of treatment $(P>$ $0.05)$, but after $28 \mathrm{~d}$ the SF in group A was higher than that in group $\mathrm{B}(P<0.05)$. Thereafter, the improvement of SF was much quicker in group $A$, and at d 56 the difference between the two groups was even more significant $(P<0.01)$. At d 14, the SI in group A was higher than in group B $(P<0.05)$ and this persisted until $56 \mathrm{~d}$ after the start of oral iron treatment, when the follow-up of this research was finished (Table 1). 


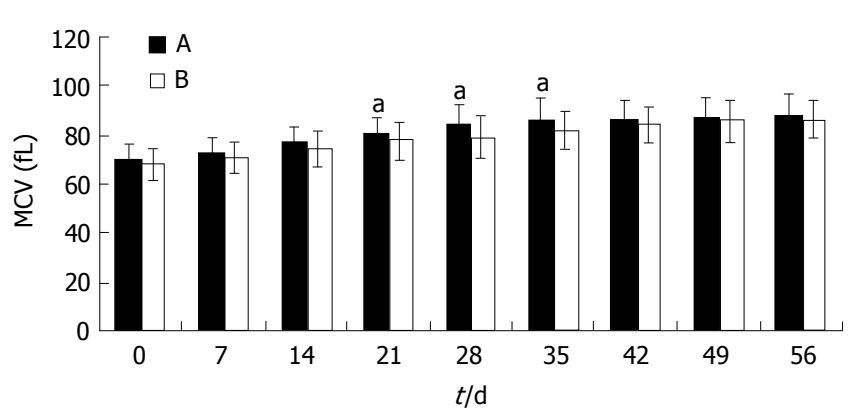

Figure 2 MCV during iron treatment (mean $\pm S D, n=43 ;{ }^{a} P<0.05$ vs group $B$ ).

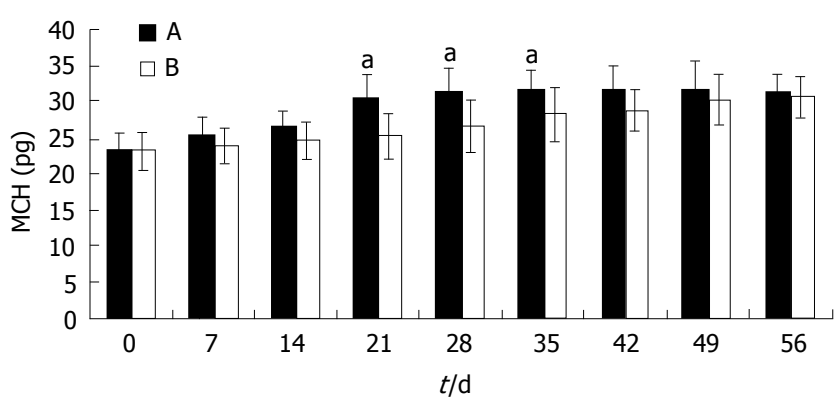

Figure $3 \mathrm{MCH}$ during iron treatment (mean $\pm \mathrm{SD}, n=43 ;{ }^{\mathrm{a}} P<0.05$ vs group $\mathrm{B}$ ).

Table 1 Alterations of SI ( $\mu \mathrm{mol} / \mathrm{L}$ ) and SF ( $\mu \mathrm{g} / \mathrm{L}$ ) during iron treatment (mean $\pm \mathrm{SD}, n=43$ )

\begin{tabular}{|c|c|c|c|c|c|c|c|c|c|c|}
\hline \multirow[t]{2}{*}{ Groups } & \multicolumn{2}{|c|}{ d 0} & \multicolumn{2}{|c|}{ d 14} & \multicolumn{2}{|c|}{ d 28} & \multicolumn{2}{|c|}{ d 42} & \multicolumn{2}{|c|}{ d 56} \\
\hline & SI & SF & SI & SF & SI & SF & SI & SF & SI & SF \\
\hline A & $3.2 \pm 1.1$ & $7.2 \pm 1.6$ & $11.3 \pm 3.2^{\mathrm{a}}$ & $9.4 \pm 2.6$ & $13.2 \pm 3.4^{\mathrm{a}}$ & $11.3 \pm 2.6^{\mathrm{a}}$ & $18.2 \pm 3.6^{\mathrm{a}}$ & $18.2 \pm 5.3^{\mathrm{a}}$ & $23.2 \pm 4.7^{\mathrm{a}}$ & $38.5 \pm 9.2^{\mathrm{a}}$ \\
\hline B & $3.3 \pm 1.2$ & $7.5 \pm 1.8$ & $8.9 \pm 2.9$ & $8.8 \pm 2.4$ & $9.2 \pm 2.2$ & $9.6 \pm 2.7$ & $13.2 \pm 2.9$ & $13.6 \pm 3.2$ & $18.2 \pm 3.7$ & $21.6 \pm 5.6$ \\
\hline
\end{tabular}

${ }^{\mathrm{a}} \mathrm{P}<0.05$ vs group $\mathrm{B}$.

\section{DISCUSSION}

$H$ pylori infection has been reported to have various manifestations in adolescents and adults, including IDA. A double-blind, placebo-controlled trial performed in adolescents with IDA and $H$ pylori infection suggested that SF was significantly lower in the $H$ pylori-infected group and that $H$ pylori eradication led to resolution of iron deficiency ${ }^{[15,16]}$. A large serosurvey of $H$ pylori in adults also found that $\mathrm{SF}$ was significantly lower in adults positive for $H$ pylori $\mathrm{IgG}$ than that in non-infected controls ${ }^{[17,18]}$. Based on our medical records of adult patients with chronic gastritis, we found that IDA occurred more frequently in $H$ pylori-positive cases. The coexistence of $H$ pylori gastritis in the 86 IDA adult patients enrolled in this study was determined by endoscopy and the urease breath test. To avoid influences from other clinical factors, the subjects were selected according to strict criteria.

It has recently been shown that extension of gastritis to the corporal mucosa occurs in a higher percentage of patients with $H$ pylori infection and IDA compared with non-anemic $H$ pylori-infected controls ${ }^{[19]}$. The blood loss in chronic gastritis, and bleeding from duodenal or gastric ulcers related to $H$ pylori infection, plays an important role in the development of iron deficiency in adults. In response to $H$ pylori chronic gastric inflammation, the epithelial cells in the mucosa are damaged, leading to detachment and apoptosis. In the absence of bleeding lesions, the possible mechanisms by which $H$ pylori is involved in the development of IDA remain unclear. Preliminary studies suggest that the growth and proliferation of $H$ pylori requires iron from the host and that some $H$ pylori strains have a specific ability to interfere with iron metabolism by binding iron to their outer membrane proteins ${ }^{[20]}$. However, other studies have demonstrated that neither virulence factors such as Cag-A4 nor mutations in the bacterial genes involved in iron uptake are associated with IDA. Given the high prevalence of $H$ pylori infection, this hypothesis cannot explain why not all patients with $H$ pylori gastritis develop IDA ${ }^{[21]}$.
Besides the occult gastrointestinal bleeding and competition for dietary iron, $H$ pylori infection can affect the gastric body and initiate the development of atrophic body gastritis that can in turn cause decreased gastric acid secretion and increased intragastric $\mathrm{pH}^{[5,22]}$. H pylori infection adversely influences the composition of the gastric juice; for example, in terms of its acidity and ascorbate content, both of which are critical for normal iron absorption ${ }^{[23]}$. These findings suggest that the physiological mechanisms that are necessary for the absorption of alimentary iron in the duodenal mucosa are impaired in patients with $H$ pylori gastritis and IDA. Thus, we planned to determine the relationship between $H$ pylori infection status and indices of IDA such as the peripheral hemogram and SI. These indices were compared between group $\mathrm{A}$, which received triple therapy combined with oral iron treatment, and group B, which received oral iron treatment only.

Before the completion of $H$ pylori eradication, there were no significant differences in parameters reflecting iron status between the two groups, though the indices were slightly higher in group A. After the 2 wk triple therapy was finished, our observations indicate that the response to oral iron treatment was significantly greater in group A than in group B. Since supplementation with ascorbic acid has been commonly shown to reduce the $\mathrm{pH}$ of gastric juice thereby increasing iron absorption, its influence was not evaluated in this research. It has been clearly demonstrated in previous studies that $H$ pylori eradication can reverse the negative effect of $H$ pylori infection on iron absorption and lead to improvement of IDA in case series and in clinical trials in both children and adults ${ }^{[4,15,16]}$. Successful $H$ pylori eradication resulted first in a significant post-treatment decrease of serum gastrin and $H$ pylori $\mathrm{IgG}$ antibody titers, and then an increase in the peripheral hemogram and SI. This normalization of iron metabolism in $H$ pylori-positive patients increased the MCV and MCH to a plateau similar to the normal level.

The $H$ pylori gastritis is increasingly considered a pos- 
sible cause of IDA refractory to oral iron treatment, and eradication of $H$ pylori may be followed by an improved response to oral iron in previously refractory IDA patients ${ }^{[4,5]}$. In this study, $H$ pylori infection slowed the trend of recovery from IDA recovery, but the indices in group B still reached normal levels. In our opinion, $H$ pylori eradication is necessary for the resolution of IDA with a lower risk of recurrence. Patients with $H$ pylori infection and IDA should receive oral iron and triple therapy simultaneously.

\section{COMMENTS}

\section{Background}

Iron deficiency anemia (IDA) is often caused by gastrointestinal bleeding due to peptic ulcer and chronic gastritis. $H$ pylori infection, which has been proved to play the main role in peptic ulcer and chronic gastritis, has been implicated as a cause of IDA refractory to oral iron treatment. The role of $H$ pylori and its eradication in IDA in patients with $\mathrm{H}$ pylori-positive gastritis has been unclear.

\section{Research frontiers}

In some cases, refractory IDA is not sensitive to oral iron treatment, especially in patients with $H$ pylori-positive gastritis. It is important to elucidate the relation of IDA to $H$ pylori infection and the effect of $H$ pylori eradication on the treatment of IDA.

\section{Related publications \\ Related publications are rare.}

\section{Innovations and breakthroughs}

This study showed that the $H$ pylori infection could slow the trend of recovery from IDA and that $H$ pylori eradication is necessary for the resolution of IDA with a lower risk of recurrence. The efficacy of simultaneous oral iron and triple therapy was evaluated.

\section{Applications}

For the treatment of IDA in patients with $\mathrm{H}$ pylori-positive chronic gastritis, it is effective and necessary to eradicate $H$ pylori infection, which can lead to satisfactory recovery of this category of IDA.

\section{Terminology}

$H$ pylori infection: $H$ pylori infection is the main cause of peptic ulcer and chronic gastritis. IDA: iron deficiency anemia is generally attributed to abnormal blood loss, which is often caused by gastrointestinal bleeding due to peptic ulcer and chronic gastritis, or in women of reproductive age by increased menstrual flow and the greater requirements of pregnancy.

\section{Peer review}

This is a good review on an interesting topic with an appropriate number of patients. The major point greatly enhances the conclusions.

\section{REFERENCES}

1 Ross EM. Evaluation and treatment of iron deficiency in adults. Nutr Clin Care 2002; 5: 220-224

2 Ciacci C, Sabbatini F, Cavallaro R, Castiglione F, Di Bella S, Iovino P, Palumbo A, Tortora R, Amoruso D, Mazzacca G. Helicobacter pylori impairs iron absorption in infected individuals. Dig Liver Dis 2004; 36: 455-460

3 Ashorn M. Acid and iron-disturbances related to Helicobacter pylori infection. J Pediatr Gastroenterol Nutr 2004; 38: 137-139

4 Konno M, Muraoka S, Takahashi M, Imai T. Iron-deficiency anemia associated with Helicobacter pylori gastritis. J Pediatr Gastroenterol Nutr 2000; 31: 52-56

5 Hacihanefioglu A, Edebali F, Celebi A, Karakaya T, Senturk $\mathrm{O}$, Hulagu S. Improvement of complete blood count in patients with iron deficiency anemia and Helicobacter pylori infection after the eradication of Helicobacter pylori. Hepatogastroenterology 2004; 51: 313-315

6 Annibale B, Capurso G, Chistolini A, D'Ambra G, DiGiulio E, Monarca B, DelleFave G. Gastrointestinal causes of refractory iron deficiency anemia in patients without gastrointestinal symptoms. Am J Med 2001; 111: 439-445

7 Conrad ME, Umbreit JN. Iron absorption and transport-an update. Am J Hematol 2000; 64: 287-298

8 Schulz HU, Schürer M, Krupp S, Dammann HG, Timm J, Gessner U. Effects of acetylsalicylic acid on ascorbic acid concentrations in plasma, gastric mucosa, gastric juice and urine--a double-blind study in healthy subjects. Int J Clin Pharmacol Ther 2004; 42: 481-487

9 Capurso G, Ricci R, Panzuto F, Baccini F, Passi S, Di Giulio E, Delle Fave G, Annibale B. Intragastric ascorbic but not uric acid is depleted in relation with the increased $\mathrm{pH}$ in patients with atrophic body gastritis and $\mathrm{H}$. pylori gastritis. Helicobacter 2003; 8: 300-306

10 Park JH, Kim SY, Kim DW, Lee WG, Rhee KH, Youn HS. Correlation between Helicobacter pylori infection and vitamin $\mathrm{C}$ levels in whole blood, plasma, and gastric juice, and the $\mathrm{pH}$ of gastric juice in Korean children. J Pediatr Gastroenterol Nutr 2003; 37: 53-62

11 Furuta T, Baba S, Takashima M, Futami H, Arai H, Kajimura M, Hanai H, Kaneko E. Effect of Helicobacter pylori infection on gastric juice $\mathrm{pH}$. Scand J Gastroenterol 1998; 33: 357-363

12 Kato S, Nakayama K, Minoura T, Konno M, Tajiri H, Matsuhisa T, Iinuma K. Comparison between the 13C-urea breath test and stool antigen test for the diagnosis of childhood Helicobacter pylori infection. J Gastroenterol 2004; 39: 1045-1050

13 Zhang ZN, Shen T. Standard diagnosis and curative effect of hematologic disease. Beijing: Science Press, 1998: 10-19

14 Mirbagheri SA, Sohrabpour AA, Hasibi M, Moghimi B, Mohamadnejad M. 14C-urea breath test in patients undergoing anti-tuberculosis therapy. World J Gastroenterol 2005; 11: 1712-1714

15 Choe YH, Kim SK, Son BK, Lee DH, Hong YC, Pai SH. Randomized placebo-controlled trial of Helicobacter pylori eradication for iron-deficiency anemia in preadolescent children and adolescents. Helicobacter 1999; 4: 135-139

16 Choe YH, Kwon YS, Jung MK, Kang SK, Hwang TS, Hong YC. Helicobacter pylori-associated iron-deficiency anemia in adolescent female athletes. J Pediatr 2001; 139: 100-104

17 Milman N, Rosenstock S, Andersen L, Jørgensen T, Bonnevie O. Serum ferritin, hemoglobin, and Helicobacter pylori infection: a seroepidemiologic survey comprising 2794 Danish adults. Gastroenterology 1998; 115: 268-274

18 Parkinson AJ, Gold BD, Bulkow L, Wainwright RB, Swaminathan B, Khanna B, Petersen KM, Fitzgerald MA. High prevalence of Helicobacter pylori in the Alaska native population and association with low serum ferritin levels in young adults. Clin Diagn Lab Immunol 2000; 7: 885-888

19 Capurso G, Lahner E, Marcheggiano A, Caruana P, Carnuccio A, Bordi C, Delle Fave G, Annibale B. Involvement of the corporal mucosa and related changes in gastric acid secretion characterize patients with iron deficiency anaemia associated with Helicobacter pylori infection. Aliment Pharmacol Ther 2001; 15: 1753-1761

20 Pérez-Pérez GI, Israel DA. Role of iron in Helicobacter pylori: its influence in outer membrane protein expression and in pathogenicity. Eur J Gastroenterol Hepatol 2000; 12: 1263-1265

21 Annibale B, Negrini R, Caruana P, Lahner E, Grossi C, Bordi C, Delle Fave G. Two-thirds of atrophic body gastritis patients have evidence of Helicobacter pylori infection. Helicobacter 2001; 6: 225-233

22 Bini EJ. Helicobacter pylori and iron deficiency anemia: guilty as charged? Am J Med 2001; 111: 495-497

23 Koike T, Ohara S, Sekine H, Iijima K, Abe Y, Kato K, Toyota T, Shimosegawa T. Helicobacter pylori infection prevents erosive reflux oesophagitis by decreasing gastric acid secretion. Gut 2001; 49: 330-334 